\title{
Introduction to Stopping Time in Stochastic Finance Theory
}

\author{
Peter Jaeger \\ Siegmund-Schacky-Str. 18a \\ 80993 Munich, Germany
}

Summary. We start with the definition of stopping time according to 4], p.283. We prove, that different definitions for stopping time can coincide. We give examples of stopping time using constant-functions or functions defined with the operator max or min (defined in 6], pp.37-38). Finally we give an example with some given filtration. Stopping time is very important for stochastic finance. A stopping time is the moment, where a certain event occurs (7, p.372) and can be used together with stochastic processes (4, p.283). Look at the following example: we install a function ST: $\{1,2,3,4\} \rightarrow\{0,1,2\} \cup\{+\infty\}$, we define:

a. $\operatorname{ST}(1)=1, \operatorname{ST}(2)=1, \operatorname{ST}(3)=2, \operatorname{ST}(4)=2$.

b. The set $\{0,1,2\}$ consists of time points: $0=$ now, $1=$ tomorrow, $2=$ the day after tomorrow.

We can prove:

c. $\{\mathrm{w}$, where $\mathrm{w}$ is Element of $\Omega$ : ST.w $=0\}=\emptyset \&\{\mathrm{w}$, where $\mathrm{w}$ is Element of $\Omega$ : ST.w $=1\}=\{1,2\} \&\{\mathrm{w}$, where $\mathrm{w}$ is Element of $\Omega$ : ST.w $=2\}=\{3,4\}$ and

$\mathrm{ST}$ is a stopping time.

We use a function Filt as Filtration of $\{0,1,2\}, \Sigma$ where Filt $(0)=\Omega_{\text {now }}$, Filt(1) $=\Omega_{f u t 1}$ and Filt $(2)=\Omega_{f u t 2}$. From a.,b. and c. we know that:

d. $\{\mathrm{w}$, where $\mathrm{w}$ is Element of $\Omega$ : ST.w $=0\}$ in $\Omega_{\text {now }}$ and

$\{\mathrm{w}$, where $\mathrm{w}$ is Element of $\Omega: \mathrm{ST} . \mathrm{w}=1\}$ in $\Omega_{f u t 1}$ and

$\{\mathrm{w}$, where $\mathrm{w}$ is Element of $\Omega$ : ST.w $=2\}$ in $\Omega_{f u t 2}$.

The sets in $d$. are events, which occur at the time points $0(=$ now $), 1(=$ tomorrow) or 2(=the day after tomorrow), see also [7], p.371. Suppose we have $\mathrm{ST}(1)=+\infty$, then this means that for 1 the corresponding event never occurs.

As an interpretation for our installed functions consider the given adapted stochastic process in the article [5].

$\mathrm{ST}(1)=1$ means, that the given element 1 in $\{1,2,3,4\}$ is stopped in 1 (=tomorrow). That tells us, that we have to look at the value $f_{2}(1)$ which is equal to 80. The same argumentation can be applied for the element 2 in $\{1,2,3,4\}$. 
$\mathrm{ST}(3)=2$ means, that the given element 3 in $\{1,2,3,4\}$ is stopped in 2 (=the day after tomorrow). That tells us, that we have to look at the value $f_{3}(3)$ which is equal to 100 .

$\mathrm{ST}(4)=2$ means, that the given element 4 in $\{1,2,3,4\}$ is stopped in 2 (=the day after tomorrow). That tells us, that we have to look at the value $f_{3}(4)$ which is equal to 120 .

In the real world, these functions can be used for questions like: when does the share price exceed a certain limit? (see [7, p.372).

MSC: 60G40 03B35

Keywords: stopping time; stochastic process

MML identifier: FINANCE4, version: 8.1.06 5.43.1297

\section{Preliminaries}

From now on $\Omega$ denotes a non empty set, $\Sigma$ denotes a $\sigma$-field of subsets of $\Omega$, and $T$ denotes a natural number.

Now we state the proposition:

(1) Let us consider a non empty set $X$, an object $t$, and a function $f$. Suppose $\operatorname{dom} f=X$. Then $\{w$, where $w$ is an element of $X: f(w)=t\}=$ $\operatorname{Coim}(f, t)$.

Proof: Set $A=\{w$, where $w$ is an element of $X: f(w)=t\} . A \subseteq$ $\operatorname{Coim}(f, t)$ by [2, (1)]. Consider $y$ being an object such that $\langle x, y\rangle \in f$ and $y \in\{t\}$.

Let $I$ be an extended real-membered set. The functor $I_{\{+\infty\}}$ yielding a subset of $\overline{\mathbb{R}}$ is defined by the term

(Def. 1) $I \cup\{+\infty\}$.

Let us note that $I_{\{+\infty\}}$ is non empty.

\section{Definition of Stopping Time}

Let $T$ be a natural number. The functor $\bigcup_{t \in \mathbb{N}: 0 \leqslant t \leqslant T}\{t\}$ yielding a subset of $\mathbb{R}$ is defined by the term

(Def. 2) $\{t$, where $t$ is an element of $\mathbb{N}: 0 \leqslant t \leqslant T\}$.

Let us note that $\bigcup_{t \in \mathbb{N}: 0 \leqslant t \leqslant T}\{t\}$ is non empty.

The functor $T_{\{+\infty\}}$ yielding a subset of $\overline{\mathbb{R}}$ is defined by the term

(Def. 3) $\bigcup_{t \in \mathbb{N}: 0 \leqslant t \leqslant T}\{t\} \cup\{+\infty\}$. 
Let us note that $T_{\{+\infty\}}$ is non empty.

In the sequel $T_{1}$ denotes an element of $T_{\{+\infty\}}, M F$ denotes a filtration of $\bigcup_{t \in \mathbb{N}: 0 \leqslant t \leqslant T}\{t\}$ and $\Sigma$, and $k, k_{1}, k_{2}$ denote functions from $\Omega$ into $T_{\{+\infty\}}$.

Let $T$ be a natural number, $F$ be a function, and $R$ be a binary relation. We say that $R$ is StoppingTime $(F, T)$ if and only if

(Def. 4) for every element $t$ of $\bigcup_{t \in \mathbb{N}: 0 \leqslant t \leqslant T}\{t\}, \operatorname{Coim}(R, t) \in F(t)$.

Let $\Omega$ be a non empty set, $M F$ be a function, and $k$ be a function from $\Omega$ into $T_{\{+\infty\}}$. Let us observe that $k$ is StoppingTime $(M F, T)$ if and only if the condition (Def. 5) is satisfied.

(Def. 5) for every element $t$ of $\bigcup_{t \in \mathbb{N}: 0 \leqslant t \leqslant T}\{t\},\{w$, where $w$ is an element of $\Omega$ : $k(w)=t\} \in M F(t)$.

Now we state the proposition:

(2) $\quad k$ is StoppingTime $(M F, T)$ if and only if for every element $t$ of $\bigcup_{t \in \mathbb{N}: 0 \leqslant t \leqslant T}\{t\},\{w$, where $w$ is an element of $\Omega: k(w) \leqslant t\} \in M F(t)$.

Proof: If $k$ is StoppingTime $(M F, T)$, then for every element $t$ of $\bigcup_{t \in \mathbb{N}: 0 \leqslant t \leqslant T}\{t\},\{w$, where $w$ is an element of $\Omega: k(w) \leqslant t\} \in M F(t)$ by 1 , (8), (12), (13)], [8, (21)]. For every element $t$ of $\bigcup_{t \in \mathbb{N}: 0 \leqslant t \leqslant T}\{t\},\{w$, where $w$ is an element of $\Omega: k(w)=t\} \in M F(t)$ by [1, (13)], [8, (22), (24)], [1, (22)].

\section{Examples of Stopping Times}

Now we state the proposition:

(3) $\Omega \longmapsto T_{1}$ is StoppingTime $(M F, T)$.

Proof: Set $c=\Omega \longmapsto T_{1}$. For every element $t$ of $\bigcup_{t \in \mathbb{N}: 0 \leqslant t \leqslant T}\{t\},\{w$, where $w$ is an element of $\Omega: c(w)=t\} \in M F(t)$ by [9, (7)], [8, (5), (4)].

Let us consider $\Omega, T, k_{1}$, and $k_{2}$. The functor $\max \left(k_{1}, k_{2}\right)$ yielding a function from $\Omega$ into $\overline{\mathbb{R}}$ is defined by

(Def. 6) for every element $w$ of $\Omega$, it $(w)=\max \left(k_{1}(w), k_{2}(w)\right.$ ).

The functor $\min \left(k_{1}, k_{2}\right)$ yielding a function from $\Omega$ into $\overline{\mathbb{R}}$ is defined by

(Def. 7) for every element $w$ of $\Omega, i t(w)=\min \left(k_{1}(w), k_{2}(w)\right)$.

Now we state the propositions:

(4) Suppose $k_{1}$ is StoppingTime $(M F, T)$ and $k_{2}$ is StoppingTime $(M F, T)$. Then there exists a function $k_{3}$ from $\Omega$ into $T_{\{+\infty\}}$ such that

(i) $k_{3}=\max \left(k_{1}, k_{2}\right)$, and

(ii) $k_{3}$ is StoppingTime $(M F, T)$. 
Proof: Set $k_{3}=\max \left(k_{1}, k_{2}\right) . k_{3}$ is a function from $\Omega$ into $T_{\{+\infty\}}$ by 2 , (3)], [3, (2)]. $k_{3}$ is StoppingTime $(M F, T)$ by (2), [8, (19)].

(5) Suppose $k_{1}$ is StoppingTime $(M F, T)$ and $k_{2}$ is StoppingTime $(M F, T)$. Then there exists a function $k_{3}$ from $\Omega$ into $T_{\{+\infty\}}$ such that

(i) $k_{3}=\min \left(k_{1}, k_{2}\right)$, and

(ii) $k_{3}$ is StoppingTime $(M F, T)$.

Proof: Set $k_{3}=\min \left(k_{1}, k_{2}\right) . k_{3}$ is a function from $\Omega$ into $T_{\{+\infty\}}$ by 2 , (3)], [3, (2)]. $k_{3}$ is StoppingTime $(M F, T)$ by (2), [,$\left.(3)\right]$.

Let $t$ be an object. The special element of $t_{\{+\infty\}}$ yielding an element of $2_{\{+\infty\}}$ is defined by the term

(Def. 8) $\operatorname{IFIN}(t,\{1,2\}, 1,2)$.

Now we state the proposition:

(6) Suppose $\Omega=\{1,2,3,4\}$. Let us consider a filtration $M F$ of $\bigcup_{t \in \mathbb{N}: 0 \leqslant t \leqslant 2}\{t\}$ and $\Sigma$. Suppose $M F(0)=\Omega_{\text {now }}$ and $M F(1)=\Omega_{f u t 1}$ and $M F(2)=\Omega_{f u t 2}$. Then there exists a function $S$ from $\Omega$ into $2_{\{+\infty\}}$ such that

(i) $S$ is StoppingTime $(M F, 2)$, and

(ii) $S(1)=1$, and

(iii) $S(2)=1$, and

(iv) $S(3)=2$, and

(v) $S(4)=2$, and

(vi) $\{w$, where $w$ is an element of $\Omega: S(w)=0\}=\emptyset$, and

(vii) $\{w$, where $w$ is an element of $\Omega: S(w)=1\}=\{1,2\}$, and

(viii) $\{w$, where $w$ is an element of $\Omega: S(w)=2\}=\{3,4\}$.

Proof: Define $\mathcal{U}$ (element of $\Omega$ ) $=$ the special element of $\$_{1\{+\infty\}}$. Consider $f$ being a function from $\Omega$ into $2_{\{+\infty\}}$ such that for every element $d$ of $\Omega$, $f(d)=\mathcal{U}(d)$ from [3, Sch. 4]. $f(1)=1$ and $f(2)=1$ and $f(3)=2$ and $f(4)=2 . f$ is StoppingTime $(M F, 2)$ and $\{w$, where $w$ is an element of $\Omega$ : $f(w)=0\}=\emptyset$ and $\{w$, where $w$ is an element of $\Omega: f(w)=1\}=\{1,2\}$ and $\{w$, where $w$ is an element of $\Omega: f(w)=2\}=\{3,4\}$ by [1, (9)], [8, (4)], [5, (24)].

\section{REFERENCES}

[1] Grzegorz Bancerek. The fundamental properties of natural numbers Formalized Mathematics, 1(1):41-46, 1990.

[2] Czesław Byliński. Functions and their basic properties. Formalized Mathematics, 1(1): 55-65, 1990. 
[3] Czesław Byliński. Functions from a set to a set. Formalized Mathematics, 1(1):153-164, 1990.

[4] Hans Föllmer and Alexander Schied. Stochastic Finance: An Introduction in Discrete Time, volume 27 of Studies in Mathematics. de Gruyter, Berlin, 2nd edition, 2004.

[5] Peter Jaeger. Modelling real world using stochastic processes and filtration. Formalized Mathematics, 24(1):1-16, 2016. doi 10.1515/forma-2016-0001.

[6] Achim Klenke. Wahrscheinlichkeitstheorie. Springer-Verlag, Berlin, Heidelberg, 2006.

[7] Jürgen Kremer. Einführung in die diskrete Finanzmathematik. Springer-Verlag, Berlin, Heidelberg, New York, 2006.

[8] Andrzej Nędzusiak. $\sigma$-fields and probability, Formalized Mathematics, 1(2):401-407, 1990.

[9] Andrzej Trybulec. Binary operations applied to functions, Formalized Mathematics, 1(2): 329-334, 1990.

Received June 27, 2017

The English version of this volume of Formalized Mathematics was financed under agreement 548/P-DUN/2016 with the funds from the Polish Minister of Science and Higher Education for the dissemination of science. 